\title{
Efficient and effective learning for safety from incidents
}

\author{
Roland Akselsson ${ }^{\mathrm{a}, \mathrm{b}, \mathrm{c}, *}$, Anders Jacobsson $^{\mathrm{a}, \mathrm{b}}$, Marcus Börjesson ${ }^{\mathrm{d}}$, Åsa Ek ${ }^{\mathrm{a}, \mathrm{b}, \mathrm{c}}$ and Ann Enander ${ }^{\mathrm{d}}$ \\ ${ }^{a}$ Ergonomics and Aerosol Technology, Department of Design Sciences, Lund University, Box 118, SE-22100 \\ Lund, Sweden \\ ${ }^{\mathrm{b}}$ Lund University Centre for Risk Assessment and Management (LUCRAM), Lund University, Box 118, SE-22100 \\ Lund, Sweden \\ ${ }^{\circ}$ Centre for Medicine and Technology for Working Life and Society (METALUND), Lund University, Box 118, \\ SE-22100 Lund, Sweden \\ ${ }^{\mathrm{d}}$ Department of Leadership and Management, Swedish National Defence College Karlstad, Våxnäsgatan 10, \\ SE-651 80 Karlstad, Sweden
}

\begin{abstract}
Learning from incidents is important for improving safety. Many companies spend a great deal of time and money on such learning procedures. The objectives of this paper are to present some early results from a project aimed at revealing weaknesses in the procedures for learning from incidents and to discuss improvements in these procedures, especially in chemical process industries. The empirical base comes from a project assessing organizational learning and the effectiveness of the different steps of the learning cycle for safety and studying relations between safety-specific transformational leadership, safety climate, trust, safety-related behavior and learning from incidents. The results point at common weaknesses in the organizational learning, both in the horizontal learning (geographical spread) and in vertical learning (double-loop learning). Furthermore, the effectiveness in the different steps of the learning cycle is low due to insufficient information in incident reports, very shallow analyses of reports, decisions that focus at solving the problem only at the place where the incident took place, late implementations and weak solutions. Strong correlations with learning from incidents were found for all safety climate variables as well as for safety-related behaviors and trust. The relationships were very strong for trust, safety knowledge, safety participation and safety compliance.
\end{abstract}

Keywords: Learning from incidents, organizational learning, safety leadership, safety climate

\section{Introduction}

Learning from incidents is considered to be an important activity for safety. Many companies also spend a considerable amount of money and time on learning from incidents. Important questions for a company are then if their learning procedures are efficient (cost effective) and effective (learning what can be learned from incidents), and if not, how can they be improved.

In a project "Learning from incidents for improving safety within dangerous operations", LINS, investigations were made in six process industries in Sweden. Methods were developed for assessing the level of learning from incidents ${ }^{1}$ and the effectiveness of the cycle for learning from incidents in an organization $^{2}$. Furthermore, a questionnaire for assessing the safety climate and the leadership style was developed. These instruments were applied at the industries revealing weaknesses in the learning process. Another goal was to find relations between safety climate, leadership style and learning from incidents.

The companies shared many weaknesses in common in their learning procedures, even if the degree of the weaknesses differed. Similar weaknesses are believed to be common in many other process industries in Sweden and in other countries with a similar

\footnotetext{
* Corresponding author. Tel. no. +46 46222 9266, fax no. +46 46222 4431, e-mail: roland.akselsson@design.lth.se
} 
culture. Thus learning from such weaknesses should be of general interest.

The objective of this paper is to present some early results from a project aimed at revealing weaknesses in the procedures for learning from incidents and to discuss improvements in these procedures.

\section{Method}

In a research project, supported by the Swedish Civil Contingencies Agency (MSB), a goal was to learn how to improve learning for safety from incidents and develop procedures for improvements. In six process industries more than 1900 incident reports were analyzed, and audits including about 180 interviews were done. Also safety climate and leadership style were investigated in the six companies.

\subsection{A tool for assessing the level of learning}

Jacobsson et al. ${ }^{1}$ developed a method for evaluating learning from incidents. Focus was on the use of double-loop learning and on the geographic spread of lessons learned. A table is used for assessing the learning from an incident at a level from 0 to $\mathrm{V}$. The table contains characteristics defining each level using the geographic spread of the learning (here horizontal learning), the highest organizational level changing governing variables (here vertical level) and the input to organizational memories.

Each incident report from 2007 and 2008 was analyzed and classified (for one company with fewer reports the period was extended and all reports from 2007 to mid-2010 were included). Then for each report also the possible learning - the potential level of learning - was estimated. In order to obtain a figure on how much the potential learning was used the ratio between the mean value of the actual level of learning and the potential level of learning was calculated for each company. We call these figures "level of learning ratios". They varied between 36 and $86 \%$. These figures do not take 'non-reported incidents' into consideration.

\subsection{A tool for assessing the effectiveness of the cycle of learning}

The learning from incidents in an organization can be divided into five steps: reporting, analysis and evaluation (assessment), decision-making, implementation and follow-up. Follow-up includes checking if
Table 1

Minimum, median and maximum of the effectiveness in the different learning steps for the six companies (Scale 1 to 10). Based on incident reports from 2007 and 2008 (for one company 2007-mid 2010)

\begin{tabular}{|lcrc|}
\hline Learning step & minimum & median & maximum \\
\hline Reporting & 3.3 & 4.4 & 7.3 \\
Analysis & 2.8 & 4.4 & 7.5 \\
Decision-making & 3.3 & 4.9 & 8.1 \\
Implementation & 4.4 & 5.5 & 8.7 \\
\hline
\end{tabular}

the implementation was successful, and applying remedies if not. Important for the cycle is also feedback to the actors in the different steps and storing in organizational memory ${ }^{3}$. Jacobsson et al. ${ }^{2}$ developed a method for assessment of the steps in the (organizational) learning cycle. Table 1 shows minimum, median and maximum values scored for the first four steps for the six investigated organizations. For the different steps in the learning cycle, reporting, assessment, decision-making and implementation the rating varied between 2.8 and 8.7 with a mean value 5,0 on a $0-10$ scale. As can be seen the span is quite large and the median is low within this interval, indicating that for most of the organizations there are potential improvements to be made.

\subsection{Linking safety climate, leadership and safety-related behaviors}

Earlier studies ${ }^{4,5}$ have shown that transformational leadership aimed towards safety seems to be effective in influencing safety climate in a positive way. A goal in the LINS project was to further study such relations. A questionnaire for studying relationships of different leadership behaviors and different dimensions of safety climate and to examine the relative impact of these dimensions on learning from incidents was developed ${ }^{6}$. A seven-factor solution measuring safety climate was found: safety communication, safety practice, management commitment, employee involvement, safety education procedures, safety education quality and priority, and reporting. Three other factors were measuring safety-related behaviors: safety compliance, safety participation and safety knowledge. Finally one factor was measuring trust between employees and management. The questionnaire was distributed to the six companies. 434 employees completed the questionnaire (73\% response rate). Measurements of the learning from 


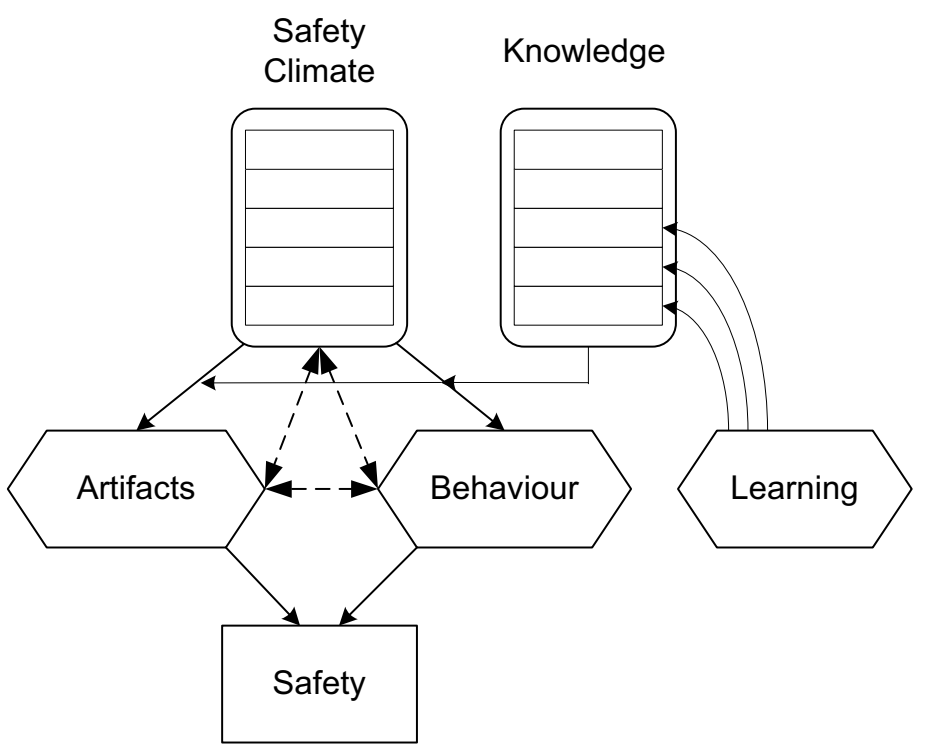

Figure 1. Learning and safety. The dashed two-ways arrows show Bandura's model for reciprocal interaction, indicating that improvements in one of the boxes safety climate, behavior and artifacts will have positive effects on the others. The multilevel boxes safety climate and knowledge signify that climate and knowledge are important at all hierarchical levels of an organization. Thus learning should affect all levels.

incidents were done by using the methods described above. Preliminary data analyses have been done using a multi-level approach.

\section{A tentative model connecting learning with safety}

In practice safety culture is a difficult concept. It is perceived as the driving force for what we are doing here and how we do it. It is also thought of as what is measured with safety culture questionnaires. Also the difference between safety culture and safety climate might be difficult to understand in practice. In this paper we define safety culture as attitudes, values and understandings about safety shared in the organization, and safety climate as a snapshot of the culture which can vary with time more rapidly than the culture. Safety climate is then what can be measured by questionnaires.

Safety behavior and safety artifacts such as the safety management system are expressions of the culture and the climate.

According to a model by Bandura ${ }^{7,8,9}$ there are reciprocal interactions between safety climate, artifacts (such as the safety management system and the manmachine interface) and behavior - see figure 1. In the figure we are also using Rasmussen's hierarchical model ${ }^{6}$ to stress the importance of the safety climate and safety knowledge at all levels. Thus, according to this model, learning improving behavior or artifacts will also improve the other components in the triangle climate, behavior, artifacts. The coupling to safety is obvious, since learning for safety in the long run will improve safety by definition. Of course learning could be wrong, but with continuous learning this will normally be detected and corrected.

According to Rasmussen ${ }^{10}$ an organization needs goal management and feedback between each level in his hierarchical model in order to act quickly in response to upcoming situations - against environmental stress. Thus it is important that the learning process involves all levels. In figure 1 we have just marked the levels within an organization. This will be further stressed below when discussing organizational learning, especially 'vertical' learning or double-loop learning. 


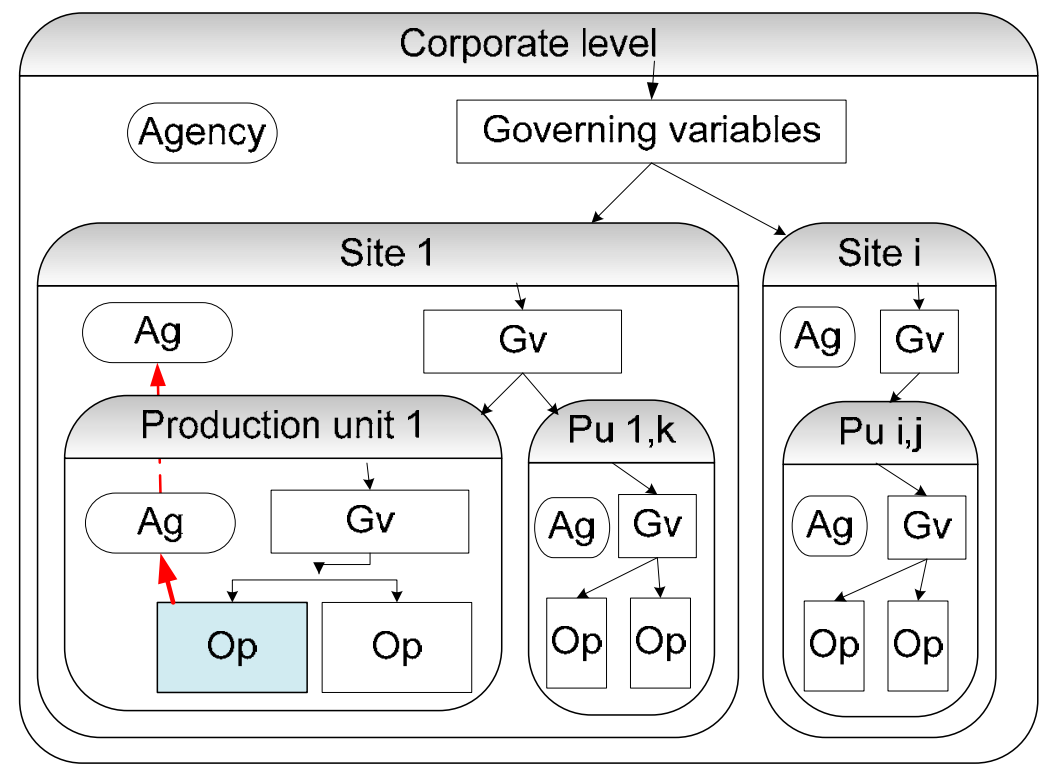

Figure 2. Vertical and horizontal organizational learning. Op = operator; $\mathrm{Ag}=($ learning $)$ agency; $\mathrm{Gv}=$ governing variables; $\mathrm{Pu}=$ production unit. The operator (light blue in the figure) discovers and, often together with a supervisor, reports an incident. The learning agency at the production unit level solves the problem without changing the governing variables for the operator (single-loop learning) or by changing the parameters (double loop learning for the operator). The agency should also decide if the problem, with its solution if solved, should be lifted to the site level for spreading to other production units or for finding better solutions with or without changing the governing variables the production units have to obey. The agency at the site level should work in a similar way.

\section{Results and Discussions}

\subsection{Level of learning - vertical and horizontal learning}

The values of the level of learning ratio (LLR) have of course a considerable uncertainty, but should give an indication on the effectiveness of the learning. An LLR of $100 \%$ as measured in this way is not the optimum since the last percent may cost more to achieve than is gained in benefit. The suggested way forward when the LLR is low is to go back and study those reports where the difference between actual and potential learning is large. A question is then what the difference comes from - lack in vertical or horizontal learning or in input to the organizational memory.

The LLR varied between 36 and $86 \%$. It was common that measures were taken to avoid a new incident or accident only at the place where the reported incident occurred, even though there was a need for similar measures in other parts of the pro- duction unit or in other production units. Furthermore, it was common that the fixes were focused on symptoms. The system weaknesses behind the reported incident were not addressed.

Figure 2 could be helpful in developing an organization and procedures for organizational learning both vertical and horizontal. Each organization should make up its own organization plan including the learning agencies. Learning agencies are very important for organizational learning ${ }^{3}$. The learning agency should possess the skill to judge when more efficient solutions could be found provided that the analysis and decision-making are lifted to higher levels in the organization, where governing parameters could be influenced (double-loop learning). The learning agency should also possess good knowledge about the production system in different production units and different sites of the organization in order to initiate geographic spread (horizontal learning).

A weakness with the level of learning ratios is that they do not take into consideration that the learning lessons from different reports could be the same. 


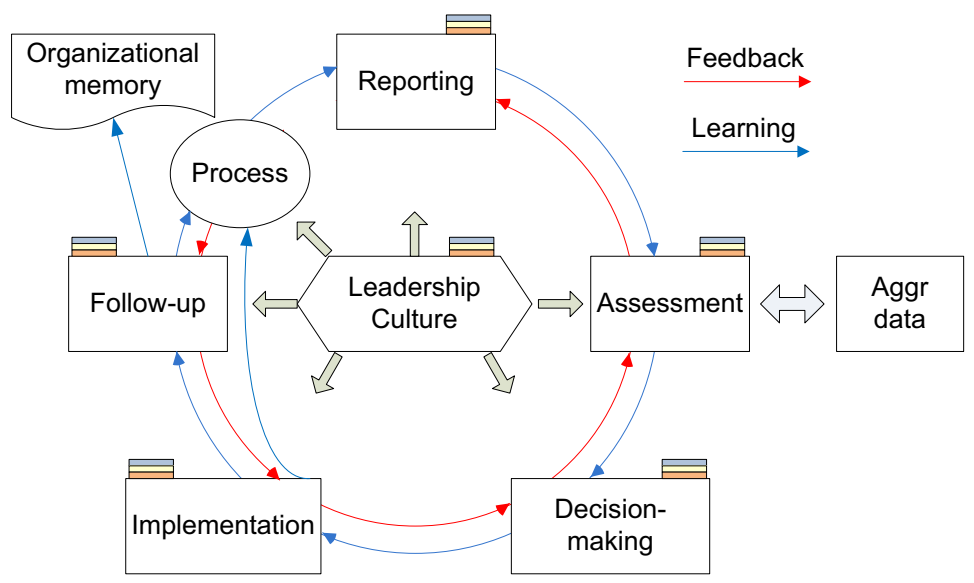

Figure 3. The cycle for organizational learning. The cycle includes the steps reporting, assessment (analysis and evaluation), decision-making, implementation and follow-up. Reports are classified after certain parameters such as type of accident, operation, and causes. The aggregated data (Aggr data) from a certain period, e.g. a year, are tracked in order to detect trends showing safety weaknesses. The colored bars indicate the involvement of all hierarchical levels of the organization. Also important, as pointed out in the paper, are leadership and safety culture.

Thus, by making deeper analyses of some reports getting a level of learning ratio close to $100 \%$ for some reports - would make it unnecessary to analyze some other reports. However, then the analyst has to identify which of this do not convey new lessons, and that requires skill. It should be possible to develop procedures for the analyst to quickly recognize reports with similar learning content and to verify that this is the case. These procedures should probably require training of reporters and improved procedures for reporting. These should make learning more efficient. Also the deeper involvement of operators should improve the safety climate and thus safety.

\subsection{The learning cycle}

Figure 3 shows the cycle for organizational learning used in this work. As seen in Table 1 all the steps, reporting, assessment, decision-making and implementation, score low.

\subsubsection{Reporting}

For reporting the main problem was that the reports were too brief telling only what happened and the injuries and damages. Information helping to reveal causes was very often lacking. Especially in one case the number of written reports per year and employee was low. This low number was in some way somewhat compensated by oral reporting, resulting sometimes in measures to eliminate or decrease the probability for recurrence.

Threshold for reporting and hidden numbers

The number of incidents reported per year is dependent on the threshold for reporting, the willingness to report and, of course, the number of incidents.

In his thesis Jacobsson ${ }^{11}$ discusses the threshold and hidden numbers. He found that the reporting frequency varied between 0.1 and 2.3 reports per employee and year for the six companies. He also found, using an expert group and judgments from the companies, that a reasonable value for the number of reportable incidents is 3 , although with large uncertainties.

Jacobsson also presented a tool for assessing the threshold for reporting including 5 types of criteria. The criteria were based on actual personal injury, actual loss of containment, the event plus one or more circumstances that could have led to an accident, deviations from procedures plus one or more circumstances that could have led to an accident and other deviations that together with one or more circumstances could have led to an accident. In the six companies the type 1 and type 2 criteria were used. Events and deviations that could have led to accidents under other circumstances and that could reveal latent conditions and weak or missing barriers were usually not reported.

The magnitude of the injury or damage may not be the best basis for determining whether an event 
should be reported. An event or a deviation that together with one or more circumstances could have led to an accident could reveal the same information about system weaknesses. And that is before an actual accident with losses has occurred. The problem is to select events and disturbances that contain the best information. That requires knowledge and motivation from the operators. More research is needed to find out how this can be achieved. Thus today for the process industry the use of criteria mainly of type 1 and 2 may be understandable but not effective.

\subsubsection{Assessment, decision-making and implementation}

The analyses were often very shallow. The analysts looked for technical defects and stopped when these were found. This, of course, led to a shallow basis for decision-making.

Decisions taken were often for local quick fixes. It would be an improvement if the decision-makers asked the following questions: Are there similar problems in other parts of our organization that should be acted on? Is the problem an indication of more general problems, such as lack of training, poor procedures, etc., that are more efficient to act on?

The formal decisions are often formulated in a general way such as finding and implementing a good solution. In the implementation phase often quick fixes are made, perhaps due primarily to costs and lack of time. Also the implementation is often delayed, thereby sending a message to the operators that safety does not have high priority.

\subsubsection{Other remarks}

The companies had weak or no procedures for handling of aggregated data to identify accumulation of incidents with similar causes and trends in the occurrence of certain types of incidents.

The low scores given to the different steps indicate that the active support from the management may not be sufficient. The low scores also indicate a weakness in the safety culture.

\subsection{Leadership, safety climate and learning from incidents}

Preliminary analyses of the questionnaire answers highlighted the importance of safety-specific transformational leadership among supervisors for especially trust, safety-related behaviors, safety knowledge and the safety climate dimensions safety communication and employee involvement ${ }^{6}$.
Positive correlations were also found between on one side safety climate dimensions, trust and safetyrelated behaviors and on the other side learning from incidents. Highest correlations with learning from incidents were found for safety knowledge safety participation, safety compliance and trust.

\section{Some key requirements for efficient and effective learning from incidents}

A goal with the ongoing analysis of the empirical material from the LINS project is to list key requirements for learning from incidents highlighted in the project, i.e. especially for chemical process industries. Below is an excerpt of the preliminary list.

- Safety culture including a just culture

- Safety-specific transformational leadership supporting the learning cycle

- Trust and safety communication

- A willingness at all hierarchical levels to contribute to cost-effective safety

- Safety knowledge in each step of the learning cycle. Training of operators/reporters on how to report based on knowledge about system weaknesses, organizational learning and the importance of the different types of thresholds

- Resources including time for each step of the learning cycle

- Clear roles in each step of the learning cycle

- Follow-up at the management level that each part of the learning cycle (Figure 3 ) is functioning including the horizontal and vertical learning. Also time between report and measure and feedback should be as short as possible

- $\quad$ Feedback (Figure 3)

- User-friendly reporting system

- Focus on learning for both process safety and occupational safety. 


\section{References}

[1] A. Jacobsson, Å. Ek, and R. Akselsson, Method for evaluating learning from incidents using the idea of "level of learning", Journal of Loss Prevention in the Process Industries 24 (2011), 333-343.

[2] A. Jacobsson, Å. Ek, and R. Akselsson, Learning from incidents - a method for assessing the effectiveness of the learning cycle. Manuscript, to be published.

[3] F. Koornneef, Organised learning from small-scale incidents, Ph.D. Dissertation, Technical University Delft, The Netherlands 2000. Retrieved August 30, 2011 from http://repository.tudelft.nl/file/

[4] J. Barling, C. Loughlin, and E.K. Kelloway, Development and test of a model linking safety-specific transformational leadership and occupational safety, Journal of Applied Psychology 87 (2002), 488-496

[5] E.K. Kelloway, J. Mullen, and L. Francis, Divergent effects of transformational and passive leadership on employee safety, Journal of Occupational Health Psychology 11 (2006), 76-86

[6] M. Börjesson and A. Enander, Safety climate, safety-specific leadership and safety-related behaviours within the Swedish process industry, in 'Lärande från incidenter för säkerhet inom farliga verksamheter [Learning from incidents for safety in dangerous operations], A. Jacobsson, M. Börjesson, Å. Ek, A. Enander, K. Petersen and R. Akselsson, eds, Rapport 1018, LUCRAM, Lund University, Lund, Sweden, 2011 (in Swedish), enclosure 6 (in English). Retrieved August 30, 2011 from http://www.lub.lu.se/en.html Search: Akselsson LINS. Paper in preparation

[7] A. Bandura, Social learning theory, Prentice-Hall, Englewood Cliffs, NJ, 1977 (from $^{12}$ )

[8] A. Bandura, Self-efficacy: toward a unifying theory of behavioural change, Psychological Review 84 (1977), 191-215 $\left(\right.$ from $^{12}$ )

[9] A. Bandura, Social foundations of thought and action: a social cognitive theory, Prentice-Hall, Englewood Cliffs, NJ, 1977 $\left(\right.$ from $^{12}$ )

[10] J. Rasmussen, Risk management in a dynamic society: a modeling problem, Safety Science 27 (1997), 183-213

[11]A. Jacobsson, Methodology for assessing learning from incidents - a process industry perspective, Ph.D. Dissertation, Lund University, Lund, Sweden, 2011.

[12] M.D. Cooper, Towards a model of safety culture, Safety Science 36 (2000), 111-136 